Corrigendum

\title{
Corrigendum to "Determination of Metals Present in Textile Dyes Using Laser-Induced Breakdown Spectroscopy and Cross-Validation Using Inductively Coupled Plasma/Atomic Emission Spectroscopy"
}

\author{
K. Rehan, ${ }^{1,2,3}$ I. Rehan, ${ }^{3}$ S. Sultana, ${ }^{4}$ M. Zubair Khan, ${ }^{3}$ Z. Farooq, ${ }^{5}$ \\ A. Mateen, ${ }^{3}$ and M. Humayun ${ }^{6}$ \\ ${ }^{1}$ State Key Laboratory of Magnetic Resonance and Atomic and Molecular Physics, \\ Wuhan Institute of Physics and Mathematics, Chinese Academy of Sciences, Wuhan 430071, China \\ ${ }^{2}$ The World Academy of Sciences (TWAS), Trieste, Italy \\ ${ }^{3}$ Department of Applied Physics, Federal Urdu University of Arts, Science and Technology, Islamabad 44000, Pakistan \\ ${ }^{4}$ Department of Chemistry, Islamia College University, Peshawar 25120, Pakistan \\ ${ }^{5}$ Department of Physics, Quaid-i-Azam University, Islamabad 45320, Pakistan \\ ${ }^{6}$ Department of Basic Sciences, University of Engineering and Technology, Peshawar 25120, Pakistan \\ Correspondence should be addressed to I. Rehan; irehanyousafzai@gmail.com
}

Received 12 October 2017; Accepted 9 November 2017; Published 22 November 2017

Copyright (C) 2017 K. Rehan et al. This is an open access article distributed under the Creative Commons Attribution License, which permits unrestricted use, distribution, and reproduction in any medium, provided the original work is properly cited.

\begin{abstract}
In the article titled "Determination of Metals Present in Textile Dyes Using Laser-Induced Breakdown Spectroscopy and Cross-Validation Using Inductively Coupled Plasma/Atomic Emission Spectroscopy" [1], the affiliation of the sixth author, A. Mateen, should be "Department of Applied Physics, Federal Urdu University of Arts, Science and Technology, Islamabad 44000, Pakistan." The corrected authors' list and affiliations are shown above.
\end{abstract}

\section{References}

[1] K. Rehan, I. Rehan, S. Sultana et al., "Determination of metals present in textile dyes using laser-induced breakdown spectroscopy and cross-validation using inductively coupled plasma/atomic emission spectroscopy," International Journal of Spectroscopy, vol. 2017, Article ID 1614654, 9 pages, 2017. 

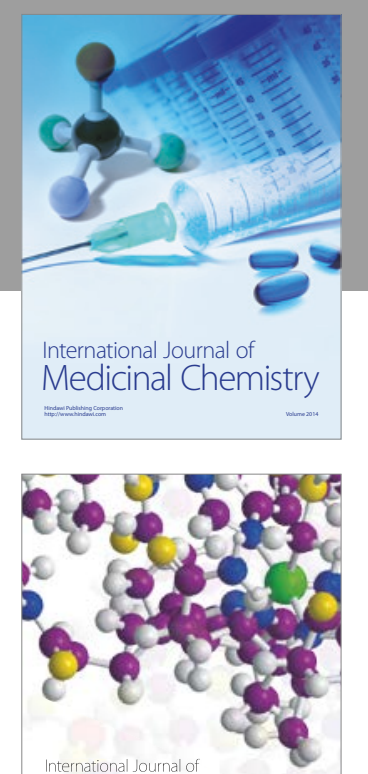

Carbohydrate Chemistry

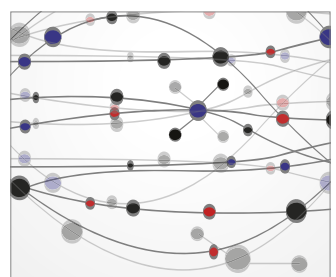

The Scientific World Journal
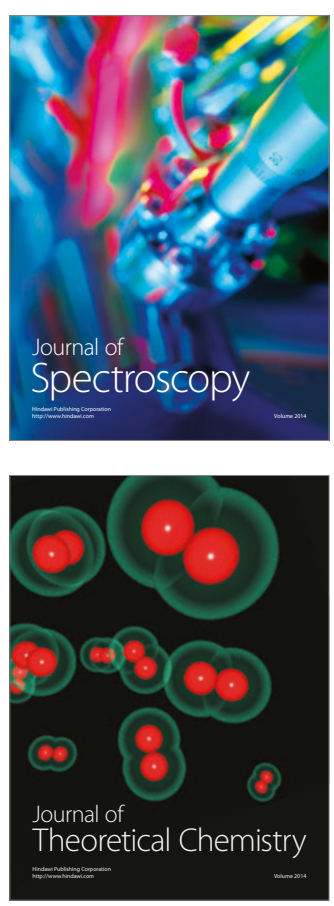
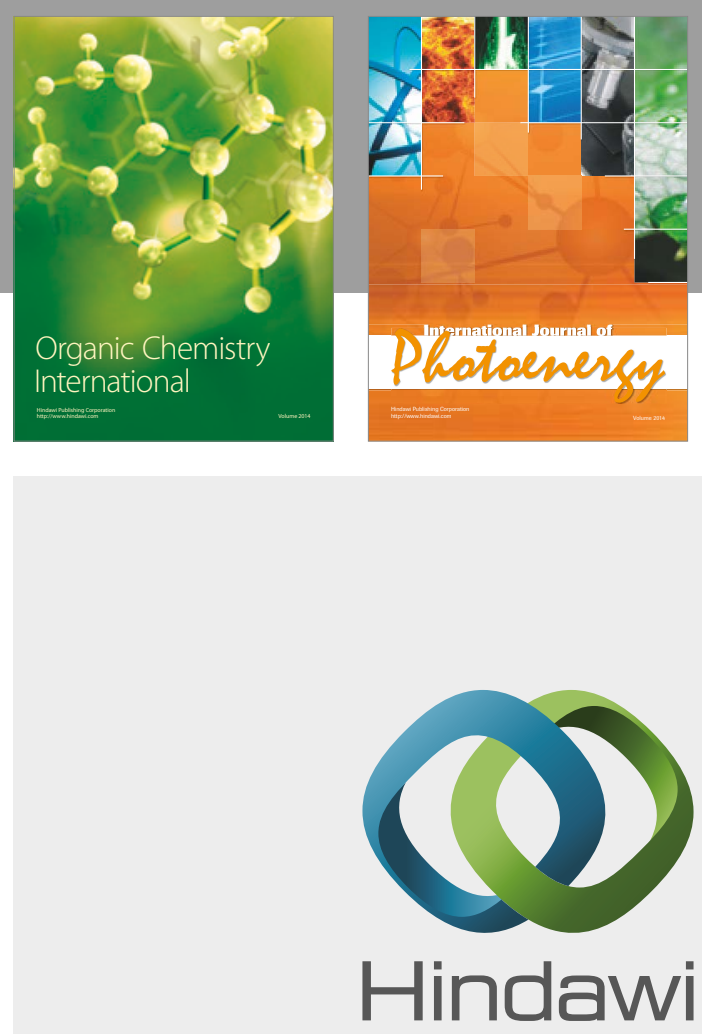

Submit your manuscripts at

https://www.hindawi.com

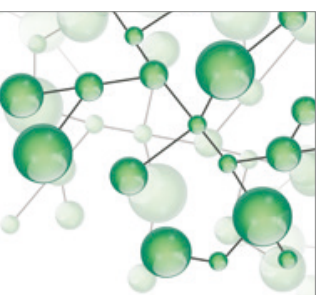

International Journal of

Inorganic Chemistry

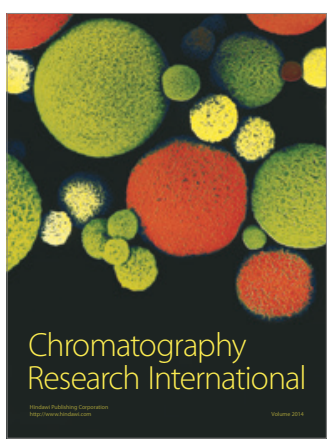

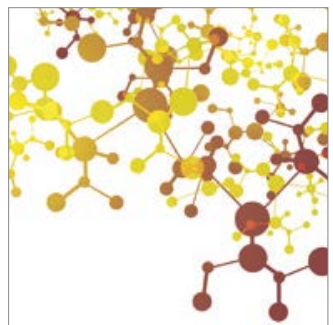

Applied Chemistry
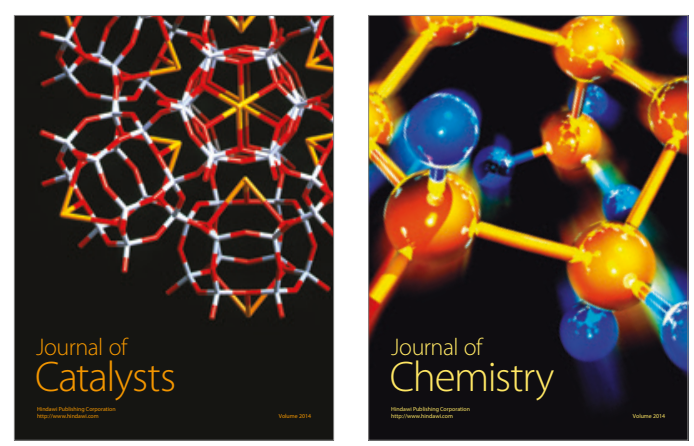
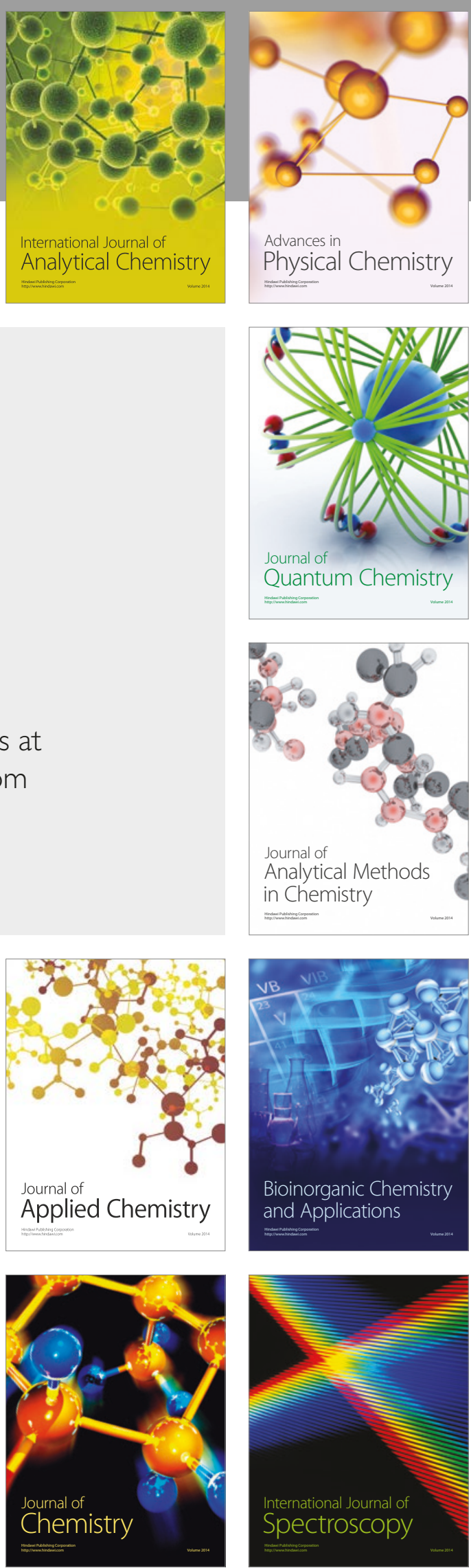